\title{
Isolation of Chlamydia trachomatis from prostatic fluid in association with inflammatory joint or eye disease
}

\author{
B T GOH,* P MORGAN-CAPNER,† AND K S LIM* \\ From the *Department of Genitourinary Medicine, King's College Hospital and the + Department of \\ Medical Microbiology, King's College Hospital Medical School, London
}

SUMMARY We describe two patients, one with peripheral arthritis, sacro-iliitis, positive HLA B27, and autoantibodies to smooth muscle and gastric parietal cell; the other with aphthoid ulcers, geographical tongue, conjunctivitis, anterior uveitis, peripheral arthralgia, and diarrhoea with distal proctocolitis. Neither patient would have been diagnosed as having urethritis on the basis of accepted microscopic criteria. In both patients, however, Chlamydia trachomatis was isolated from the prostatic fluid, but not the urethra.

\section{Introduction}

Chlamydia trachomatis has been implicated in the aetiology of the sexually transmitted form of Reiter's disease by its isolation from the urethra. ${ }^{1}$ Urethritis in patients with features suggestive of Reiter's disease or sexually acquired reactive arthritis, is investigated by the examination of Gram stained urethral smears and urine samples for polymorphonuclear leucocytes, and it may be necessary to examine an early morning urethral smear to detect low grade urethritis. A criterion for diagnosis of urethritis is ten or more polymorphonuclear leucocytes per high power field ( $\times 1000$ objective). The isolation of $C$ trachomatis in the absence of such evidence of inflammation in an early morning smear has not been reported. ${ }^{2}$

Although the diagnostic criteria are controversial, clinically silent prostatitis has been described in a high percentage of patients with Reiter's disease, anterior uveitis, ${ }^{3}$ and ankylosing spondylitis, ${ }^{45}$ and in up to $38 \%$ of apparently healthy men. ${ }^{6}$ In view of these possible associations, Gram stained smears and chlamydial culture of prostatic fluid were performed in two patients with inflammatory joint or eye disease, but in whom there was no evidence of urethritis.

Address for reprints: Dr B T Goh, Whitechapel Clinic, The London Hospital, London E1 1BB

Accepted for publication 31 May 1983

\section{Case Reports}

CASE 1

A 28 year old heterosexual man presented with progressive arthritis of both knees, the right ankle, and the left wrist and elbow of one month's duration. There was no history of conjunctivitis and no genital symptoms. Extramarital intercourse had occurred nine months previously. Polymorphonuclear leucocytes were not seen in a urethral smear taken with a plastic loop or in an early morning urethral smear. Culture of endourethral material obtained with a sterile cotton tipped wire swab introduced $4-5 \mathrm{~cm}$ into the urethra gave negative results for $C$ trachomatis. A second early morning urethral smear gave negative results, and a prostatic massage was performed using the method described elsewhere. ${ }^{7}$ The prostatic fluid showed only an occasional polymorphonuclear leucocyte on Gram stained smear, but $C$ trachomatis was isolated by a method previously described. ${ }^{8}$ The patient abstained from sexual intercourse while under investigation. Urethral smear and cultures for Neisseria gonorrhoea gave negative results. A microimmunofluorescence test $^{9}$ for chlamydial antibodies in serum showed IgG (titre of $1 / 256$ ) against the D-K serotypes. Chlamydia specific IgM was not detected. Other investigations gave the following results: erythrocyte sedimentation rate $104 \mathrm{~mm}$ in the first hour; a hypochromic, microcytic anaemia with haemoglobin concentration $10 \cdot 7$ $\mathrm{g} / \mathrm{dl}$, mean cell volume $71 \mathrm{fl}$, mean cell haemoglobin $23.6 \mathrm{pg}$, white cell count $6.4 \times 10^{9} / 1$; no rheumatoid factor, antinuclear antibody, or anti- 
mitochondrial antibody; positive results for antismooth muscle antibody, anti gastric parietal cell antibody, and HLA A2, A32, B7, and B27; results of the Venereal Diseases Research Laboratory (VDRL) test and the Treponema pallidum haemagglutination assay (TPHA) were negative; $x$ ray films of the sacroiliac joints showed right sacro-iliitis and of the knees showed bilateral small effusions.

He was treated with indomethacin $25 \mathrm{mg}$ orally three times a day and $75 \mathrm{mg}$ at night and doxycycline $200 \mathrm{mg}$ daily for 20 days with clinical improvement. Subsequent prostatic fluid culture for $C$ trachomatis was negative. Three months later, erythrocyte sedimentation rate remained high $(91 \mathrm{~mm}$ in the first hour) and his anaemia persisted. His wife was asymptomatic and no abnormality was found on genital examination. She was treated empirically with doxycycline.

\section{CASE 2}

A 19 year old homosexual man presented with pediculosis pubis, painful aphthoid ulcers of lips and buccal mucosa, and areas of depapillation on the tongue. The aphthoid ulcers resolved on treatment with Triadcortyl in Orabase (Squibb), but the geographical tongue remained. Three months later he developed bilateral conjunctivitis and anterior uveitis of the left eye with posterior synechiae. This was followed by arthralgia of the metacarpophalangeal joints, the proximal interphalangeal joints, and the wrists of both hands, left knee, and left elbow. There was only an occasional, polymorphonuclear leucocyte in an early morning urethral smear. The rectum appeared normal and a rectal smear showed no polymorphonuclear leucocytes. Cultures for $C$ trachomatis from the urethra, rectum, and conjunctivae were negative, and $N$ gonorrhoeae was not detected in smears and cultures taken from the urethra, rectum, or pharynx. A second early morning urethral smear was normal and a smear of the prostatic fluid showed no polymorphonuclear leucocytes. Chlamydial culture of the prostatic fluid, however, gave positive results, although no chlamydial antibodies were detected in the serum. Other investigations showed: erythrocyte sedimentation rate $4 \mathrm{~mm}$ in the first hour; full blood count normal; no rheumatoid factor or autoantibodies but HLA A11, A28, B12, and B41 antibodies were present; Venereal Diseases Research Laboratory (VDRL), Treponema pallidum haemagglutination (TPHA), and hepatitis B surface antigen (HBsAg) tests gave negative results; $x$ ray films of chest and joints were normal. The ocular symptoms were successfully treated with prednisolone, and chlamydial culture of the prostatic fluid gave negative results after treatment with doxycycline by mouth $200 \mathrm{mg}$ daily for $\mathbf{2 0}$ days. Seven months after his initial illness he developed abdominal pain and diarrhoea three to four times a day. The stools were mixed with mucus and occasionally tinged with blood. Examination for protozoa and culture for bacterial pathogens gave negative results. As his symptoms persisted in spite of antidiarrhoeal agents, a barium enema was performed, which showed narrowing and loss of normal haustral pattern in the rectum and sigmoid colon, and a loss of rectal curvature with granularities of the mucosal pattern in the sigmoid colon. Sigmoidoscopy up to $20 \mathrm{~cm}$ and biopsies from the rectum and sigmoid colon, however, gave results within normal limits. He was treated with oral salazopyrine $500 \mathrm{mg}$ four times daily with good clinical response. His sexual contacts were untraceable.

\section{Discussion}

Reiter's disease is difficult to define, as the manifestations are varied and often incomplete. The first patient presented with peripheral arthritis, sacroiliitis, and had the HLA B27 phenotype, which were suggestive of Reiter's disease. His positive anti smooth muscle and anti gastric parietal cell antibodies, however, together with a persistent raised erythrocyte sedimentation rate, suggest an element of autoimmune disease. The second patient suffered aphthoid ulcers, geographical tongue, conjunctivitis, anterior uveitis, peripheral arthralgia, and diarrhoea with distal proctocolitis. The differential diagnosis lies between Reiter's disease, chronic inflammatory bowel disease, and possibly Behçet's syndrome. The relation between Reiter's disease and chronic inflammatory bowel disease is uncertain, although cases of Reiter's disease with non-infective colitis have been reported. ${ }^{10} 11$ These two cases illustrate the overlapping features of Reiter's disease, other diseases in the seronegative spondarthrides, and autoimmune diseases.

In the two patients we describe, $C$ trachomatis was isolated from prostatic fluid, but not from the urethra. Indeed, there was no evidence of urethritis in these two patients, which suggests that $C$ trachomatis infection of the prostate may occur in the absence of urethritis. As only one of these patients had any observable polymorphonuclear leucocytes, and a high percentage of apparently healthy men may have excess polymorphonuclear leucocytes in the prostatic fluid, ${ }^{6}$ examination of a smear of the prostatic fluid does not seem to be helpful. Chlamydial urethritis appears to be one of the possible triggering factors in Reiter's disease, with the overall urethral isolation rate of $C$ trachomatis being as high as $39 \% .{ }^{1}$ The $C$ trachomatis in the prostatic fluid of these two patients may have been a chance association or the cause of their illnesses. 
Although little evidence has been found of an aetiological role for $C$ trachomatis in non-bacterial prostatitis, ${ }^{12}$ chlamydial antibodies are higher in patients with non-acute prostatitis than in controls. ${ }^{13}$ The first patient had chlamydial antibodies, but not of the IgM class. Specific IgM, however, has been absent in cases of proved recent chlamydial infection. ${ }^{14}$ The second patient had no detectable chlamydial antibodies. Again this is not at variance with the isolation of $C$ trachomatis, as Wang et al ${ }^{14}$ showed that $19 \%$ of men with culture positive chlamydial genital infection did not have detectable chlamydial antibodies in paired sera.

Our findings in these two patients indicate that further investigation is required into the possibility of infection of the prostate with $C$ trachomatis in the absence of urethritis and local symptoms. To elucidate the possible role of $C$ trachomatis as a triggering factor in Reiter's disease or sexually acquired reactive arthritis, we suggest that chlamydial culture of prostatic fluid is considered, even if urethral culture is negative and urethritis is absent.

We thank Dr J D Treharne of the Institute of Ophthalmology, London for performing the chlamydial serology tests.

\section{References}

1. Kousa M, Saikku P, Richmond S, Lassus A. Frequent association of chlamydial infection with Reiter's syndrome. Sex Transm Dis 1978;5:57-61.

2. Dunlop EMC. Chlamydial infection: terminology, disease, treatment, local complications and systemic disease. In: Harris JRW ed. Recent advances in sexually transmitted diseases. Edinburgh: Churchill Livingstone, 1981;101-40.

3. Catterall RD. Uveitis, arthritis and non-specific genital infection. Br J Vener Dis 1960;36:27-9.

4. Mason RM, Murray RS, Oates JK, Young AC. Prostatitis and ankylosing spondylitis. $\mathrm{Br}$ Med $J$ 1958; 1:748-51.

5. Romanus $R$. Pelvo-spondylitis ossificans in the male and genitourinary infection. Acta Med Scand 1953;145; (suppl 280): 233-41.

6. O'Shaughnessy EJ, Perrino PS, White JD. Chronic prostatitis fact or fiction? $J A M A$ 1956; $160: 540-2$.

7. King A, Nicol C, Rodin P. Venereal Diseases. 4th ed. London: Baillière Tindall, 1980:398-9.

8. Goh BT, Morgan-Capner P, Lim KS. Chlamydial screening of pregnant women in a sexually transmitted diseases clinic. $B r J$ Vener Dis 1982; 58:327-9.

9. Treharne JD, Darougar S, Jones BR. Modification of the microimmunofluorescence test to provide a routine serodiagnostic test for chlamydial infection. $J$ Clin Pathol 1977; 30:510-7.

10. Bourell M, Pawlotsky Y, Lenoir P, Simon M. Rectocolite ulcerohémorrgique et syndrome de Fiessinger-Leroy-Reiter avec cellules à inclusions. Sem Hop Paris 1968;44: 1005-8.

11. Hickling P, Martin MFR, Brooks S. A case of Reiter's disease complicated by fulminating colitis. Ann Rheum Dis 1982; 41: 189-91.

12. Mårdh P-A, Ripa KT, Colleen S, Treharne JD, Darougar $S$. Role of $C$ trachomatis in non-acute prostatitis. Br J Vener Dis 1978;54:330-4.

13. Mårdh P-A, Colleen S, Holmquist B. Chlamydia in chronic prostatitis. Br Med J 1972; 4:361.

14. Wang SP, Grayston JT, Kuo CC, Alexander ER, Holmes KK. Serodiagnosis of $C$ trachomatis infection with the microimmunofluorescence test. In: Hobson D, Holmes KK eds. Nongonococcal urethritis and related infections. Washington DC: American Society for Microbiology, 1977;237-48. 